\title{
Cilia, mitochondria, and cardiac development
}

\author{
Bill Chaudhry and Deborah J. Henderson
}

Cardiovascular Research Centre, Institute of Cenetic Medicine, Newcastle University, Newcastle Upon Tyne, United Kingdom.

\begin{abstract}
Motile cilia provide propulsion, and immotile ones are enriched with receptors. Both are required to establish left-right identity in the developing embryo and are also implicated in a wide range of human diseases. Abnormalities in cilial function underlie heterotaxy congenital heart disease (CHD) occurring in individuals with laterality disturbance. Mitochondrial function and cellular energetics, through mTOR and autophagy, are now linked with cilial function, revealing new mechanisms and candidate genes for syndromic human disease. In the current issue of the $J C I$, Burkhalter et al. ask the question: Can mitochondrial disturbances produce ciliopathy and does this explain some cases of heterotaxy?
\end{abstract}

length might link to defects in left-right patterning, they then used the zebrafish embryo, an unrivaled model for investigating left-right specification, to show that longer cilia disrupted nodal flow and randomized left-right patterning. Using genomic data, they also showed that people with heterotaxy have an increased incidence of damaging variants in mitochondrial-associated genes, and that recapitulation of these rare variants in zebrafish embryos led to lengthened cilia and left-right patterning defects.

Studies in different experimental models have indicated that shorter, fewer, or misplaced cilia disturb fluid circulation in the embryonic node and lead to randomized left-right specification (6). However, the data describing abnormally long cilia are less abundant. Cilia are actively constructed and intraflagellar transport (IFT) proteins are considered to be the main effectors of ciliary length. For example, in Chlamydomonas, overexpression of anterograde IFT proteins results in longer cilia, whereas decreased expression results in shortened cilia (7). Similarly, in mammalian cells, increasing the mobility of the anterograde IFT complex results in longer cilia. Signaling through cyclic AMP, calcium release (8), and autophagy (9) are closely linked with cilial length. It is unclear how these signaling events are integrated, but the mTOR signaling pathway is likely to be important. This evolutionarily conserved and highly complex pathway transduces environmental conditions and cellular energetic status to control cellular growth and metabolism (10), and has also been linked to mitochondrial biogenesis (11). Further studies are needed to understand whether cilial elongation is a specific manifestation of mTOR dysregulation, or if defects in other signaling pathways can also produce longer cilia, and ultimately left-right disturbance. Moreover, it remains to be seen whether mTOR signaling affects mitochondrial function and cilial length in parallel or elongates cilia through modulation of mitochondrial function. If disruption of the mito- 


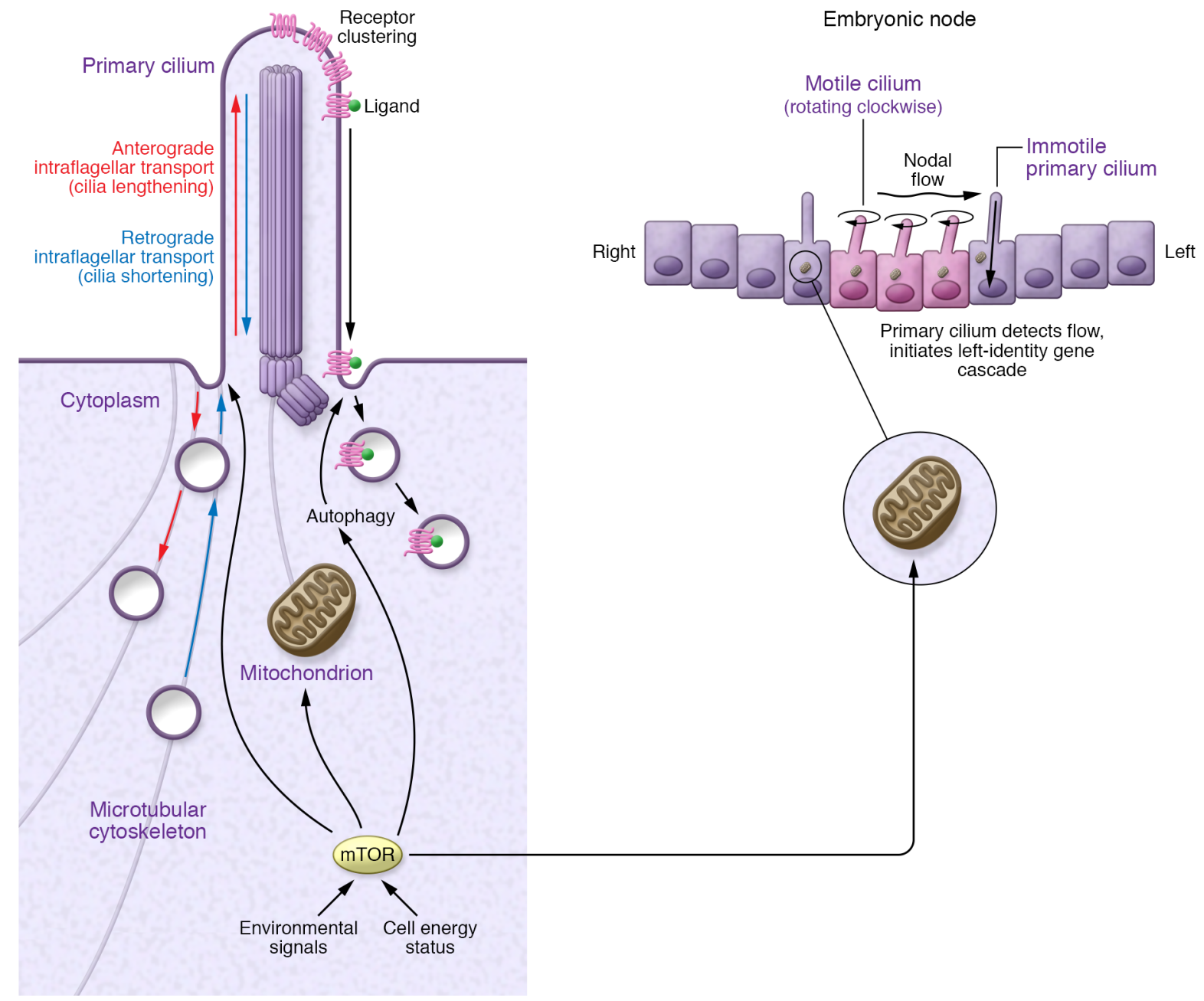

Figure 1. Aberrant mitochondrial function and cilial length. The primary cilium is actively constructed and deconstructed when the cell is not dividing. Receptors are enriched on the ciliary membrane and when bound by ligands internalized within the ciliary pocket. The same proteins used within the cilium are also used for vesicular transport across the general microtubular cytoskeleton. The mTOR pathway couples the cellular energetic status to mitochondrial biogenesis and cilial length. Cilia located in the embryonic node are also regulated by mTOR. Motile cilia rotate in a clockwise direction and create flow across the surface of the node. This is detected by primary immotile cilia at the rim of the node, activating a gene-signaling cascade resulting in the establishment of left somatic identity.

chondrial-derived energy source is sufficient to change cilial length, then this would require a differential sensitivity between the construction and dismantling processes. This is possible, as specific kinesins involved in disassembly have been identified (reviewed in ref. 7). Another difficult issue to resolve in cilial biology is that proteins involved in cilial trafficking may also be used to move cargo around the microtubular cytoskeleton, meaning some aspects of developmental disruption or disease could originate within the body of the cell (12).

\section{Cilial dysfunction and heart malformation}

The link between cilia and congenital heart disease (CHD) is well known and primarily exists via heterotaxy, or possibly syndromic CHD (13). In a recent forward genetic mouse screen, more than half of the CHD-implicated genes were ciliarelated, and many others were involved in cilia-transduced signaling pathways or cellular processes required for cilia formation or function (12). In contrast to mice, the burden of cilial dysfunction in the human CHD patient population, especially those without syndromic CHD, appears small. Large-scale genomic studies (reviewed in ref. 13) have not implicated cilial genes to any great extent in patients. Developmental biology studies have supported a role of cilia in left-right axis disturbance, but studies indicating a direct role in cardiac progenitor cells remain limited. One possible explanation for the human-mouse discrepancy is an inherent bias in gene ontology due to variations in the depth of annotation information. Well-researched areas such as cilia are likely to have more and diverse entries compared with other lessdeveloped areas such as cardiac development (14). Human patients are also survivors of development. While laterality disturbance is clearly associated with heart malformation (15), heterotaxy may not represent the full range of phenotypic possibilities. Some patterns of randomized organ placement may not be compatible with successful early embryogenesis.

A notable strength of the Burkhalter et al. study is that the conclusions are based 
on testing of logical laboratory and genomic hypotheses, and that the functional assays used are relevant in cilial function and left-right specification.

\section{The second heart field and heterotaxy}

Circumstantial evidence is starting to suggest that malformations seen in laterality disturbance could be explained by leftright patterning within the second heart field (SHF). Although initial patterning of atrial appendages is presumed to relate to their left-right orientation on the primitive heart tube, Cre-lox lineage tracing in mice suggests that both atria and appendages are mainly derived from the SHF (16). The spectrum of malformations seen in heterotaxy includes atrioventricular septal defects (AVSDs), pulmonary stenosis/ atresia (PS/A), double outlet right ventricle (DORV), and transposition of the great arteries (TGAs). All these affected structures also originate from the SHF. In the case of AVSDs, intracardiac septation requires the SHF-derived dorsal mesocardial projection (DMP). The role of cilia in the formation of the DMP has been elegantly shown both in association with a left-right disturbance but also in the absence of a laterality phenotype (17), indicating a potential local role for cilia in heart malformation. Other suggestions, including aberrant Sonic hedgehog signaling in the ventricular myocardium leading to septal defects, are less convincing, as defects only occur in the context of a grossly abnormal myocardial architecture (18). However, functional evaluation of novel genes identified through developmental biology or genomic studies may yet implicate cilia in nonsyndromic cardiovascular malformations.

\section{Barth syndrome}

Increasingly, developmental syndromes, especially those with neurological features, are linked to mitochondrial abnormalities, but there is little to suggest an increased frequency of CHD or left-right patterning disorder in these patients. It is possible that the cilia in the human embryonic node are resistant to mitochondrial dysfunction, or that the combination of left-right disorder, CHD, mitochondrial abnormalities, and cilial dysfunction are lethal to the early embryo. In this regard, the Hippo pathway effector protein TAZ is particularly interesting. Loss of function TAZ mutations cause mitochondrial insufficiency and are associated with fetal and neonatal cardiomyopathy known as Barth syndrome (19). Although aberrant cilia have not been reported in patients, mice and flies lacking TAZ are sterile with defects in the sperm, suggesting this could be a cilia-related defect. Interestingly, a link between left ventricular noncompaction, a common feature of the cardiomyopathy in Barth syndrome, and heterotaxy syndrome has been observed (20). Thus, Barth syndrome may be the survivable portion of a broader TAZopathy syndrome.

\section{Future directions}

To take these studies further, both in the laboratory and in the clinic, more accurate phenotyping, especially of the heart, is needed. The type of septal defect and detail of outflow tract malformation are of major significance to cardiac developmental biologists and essential for the correct interpretation of genomic data. Alongside this, adequately powered and reported animal studies are mandatory. In the clinic, full three-generation family histories with phenotyping of the apparently unaffected family members is essential, both to expose the full extent of these cilial conditions and to provide accurate genetic counseling. The linking of mitochondrial gene mutations to heterotaxy thus expands the range of genes that need to be considered in the context of isolated and syndromic CHD.

\section{Acknowledgments}

We would like to acknowledge the British Heart Foundation for funding our zebrafish studies relating to cilia and cardiac development (PG/15/58/31611).

Address correspondence to: Bill Chaudhry, Cardiovascular Research Centre, Institute of Genetic Medicine, Newcastle University, Central Parkway, Newcastle upon Tyne, NE1 3BZ, United Kingdom. Phone: 44.191.241.8681; Email: bill.chaudhry@ ncl.ac.uk.
1. Satir P. CILIA: before and after. Cilia. 2017;6:1.

2. Eggenschwiler JT, Anderson KV. Cilia and developmental signaling. Annu Rev Cell Dev Biol. 2007;23:345-373.

3. Grimes DT, Burdine RD. Left-right patterning: breaking symmetry to asymmetric morphogenesis. Trends Genet. 2017;33(9):616-628.

4. Reiter JF, Leroux MR. Genes and molecular pathways underpinning ciliopathies. Nat Rev Mol Cell Biol. 2017;18(9):533-547.

5. Burkhalter MD, et al. Imbalanced mitochondrial function provokes heterotaxy via aberrant ciliogenesis. JClin Invest. 2019;129(7):2841-2855.

6. Amack JD. Salient features of the ciliated organ of asymmetry. Bioarchitecture. 2014;4(1):6-15.

7. Keeling J, Tsiokas L, Maskey D. Cellular mechanisms of ciliary length control. Cells. 2016;5(1):E6.

8. Besschetnova TY, Kolpakova-Hart E, Guan Y, Zhou J, Olsen BR, Shah JV. Identification of signaling pathways regulating primary cilium length and flow-mediated adaptation. Curr Biol. 2010;20(2):182-187.

9. Pampliega $\mathrm{O}$, et al. Functional interaction between autophagy and ciliogenesis. Nature. 2013;502(7470):194-200.

10. Saxton RA, Sabatini DM. mTOR signaling in growth, metabolism, and disease. Cell. 2017;168(6):960-976.

11. Zhang Y, Xu H. Translational regulation of mitochondrial biogenesis. Biochem Soc Trans. 2016;44(6):1717-1724.

12. Li Y, Klena NT, Gabriel GC, Liu X, Kim AJ, Lemke $\mathrm{K}$, et al. Global genetic analysis in mice unveils central role for cilia in congenital heart disease. Nature. 2015;521(7553):520-524.

13. Zaidi S, Brueckner M. Genetics and genomics of congenital heart disease. Circ Res. 2017;120(6):923-940.

14. Khodiyar VK, et al. The representation of heart development in the gene ontology. Dev Biol. 2011;354(1):9-17.

15. Jacobs JP, et al. The nomenclature, definition and classification of cardiac structures in the setting of heterotaxy. Cardiol Young. 2007;17(Suppl 2):1-28.

16. Crucean A, et al. Re-evaluation of hypoplastic left heart syndrome from a developmental and morphological perspective. Orphanet J Rare Dis. 2017;12(1):138.

17. Burnicka-Turek O, et al. Cilia gene mutations cause atrioventricular septal defects by multiple mechanisms. Hum Mol Genet. 2016;25(14):3011-3028.

18. Wiegering A, Rüther U, Gerhardt C. The role of hedgehog signalling in the formation of the ventricular septum. J Dev Biol. 2017;5(4):E17.

19. Clarke SL, et al. Barth syndrome. Orphanet J Rare Dis. 2013;8:23.

20. Martinez HR, Ware SM, Schamberger MS, Parent JJ. Noncompaction cardiomyopathy and heterotaxy syndrome. Prog Pediatr Cardiol. 2017;46:23-27. 